\title{
Chiral Resolution of Naptalam Using D-tartaric acid and Functionalized Multi-walled Carbon Nanotubes
}

\author{
Dushu Huang ${ }^{1 *}$, Yunhui Long ${ }^{1}$, Wei Liu ${ }^{1}{ }^{1}$, Yong Hong ${ }^{1}$, Ming Zhong ${ }^{2}$, Shijuan \\ ${ }^{1}$ College of Science, Honghe University, Mengzi, Yunnan, China, 661199 \\ ${ }^{2}$ School of Chemistry and Chemical Engineering,Hunan Institute of Science and Technology, \\ Yueyang, Hunan, P. R. China, 414006 \\ a email: huangdushu2008@163.com
}

\begin{abstract}
Keywords: Functionalized multi-walled carbon nanotubes, Chiral resolution, Naptalam, Thin-layer Chromatography
\end{abstract}

\begin{abstract}
Chiral resolution of naptalam using various functionalized multi-walled carbon nanotubes (MWCNTs) mediated thin-lay chromatography was studied. D-tartaric acid was used as chiral selectors and cyclohexane-alcohol solvent mixture was used as developers. The results indicated that under the condition of $\mathrm{V}($ cyclohexane $): \mathrm{V}(\mathrm{n}$-butanol $)=30: 70$, the sizes of spots were even, and there is no phenomenon of tail and the effect of resolution was the best. The results indicated that chiral naptalam can be resoluted well by thin-lay chromatography impregnated with various functionalized multi-walled carbon nanotubes such as MWCNTs, aligned MWCNTs, amino functionalized MWCNTs and carboxylated MWCNTs. And the relative RF value of amino functionalized multi-walled carbon nanotubes impregnated thin-lay chromatography was significantly higher than that of other types of multi-walled carbon nanotubes impregnated thin-lay chromatography. The relative RF value of amino multi-walled carbon nanotubes mediated thin-lay chromatography was 4.32 .
\end{abstract}

\section{Introduction}

Naptalam is a selective pre-emergence herbicide. It controls many broad-leaved weeds and some grasses in cucurbits, asparagus, peanuts, soybeans and established woody ornamentals [1]. Many commercial drugs in current use contain chiral structures and thus consist of enantiomers. The biological effects of enantiomers such as toxicity, mutagenicity, carcinogencity, and endocrine disruption activity are generally different [2-7]. Therefore, resolution of chiral herbicides has attracted wide attention. Currently, chiral chromatographic separation is considered to be the most effective method of resolution due to its advantages such as fast, easy and good separation effect [8]. And selection of the chiral stationary phase is very important in chiral chromatographic separation [9-11].

Due to their unique structure and excellent properties, multi-walled carbon nanotubes have been received wide attension since it was found in the early 1990s. It has excellent properties such as heat resistance, corrosion resistance, impact resistance, heat and electrical conductivity, high temperature and high strength. The study found that carbon nanotubes are one of the materials with best mechanical properties [12-14]. It has important applications in many areas and is one of the most promising nanomaterials in the 21 st century. Aligned multi-walled carbon nanotubes arrange in the same direction and has excellent electrical conductivity and good dispersion.

Herein, a method of using multi-walled carbon nanotubes mediated thin-lay chromatography with D-tartaric acid as chiral selectors for resolution of naptalam was demonstrated. Tartaric acid, also known as dihydroxybutane-dioic acid, has been widely used in various industrial fields, especially in medicine and the food industry. D-tartaric acid and its esters are known as chiral selectors, most widely used in chiral separations, including extraction separation, chiral membrane separation and chromatographic separation, etc. The MWCNTs (outer diameter $<8 \mathrm{~nm}$, length $10-30 \mu \mathrm{m}$, purity $\geq 95 \mathrm{wt} \%$ ), aligned Multi-walled carbon nanotubes (outer diameter 10-20 nm, 
length 30-100 $\mu \mathrm{m}$, purity $\geq 95 \mathrm{wt} \%$ ), amino functionalized Multi-walled carbon nanotubes (outer diameter 8-15 $\mathrm{nm}$, length $\sim 50 \mu \mathrm{m}$, purity $\geq 95 \mathrm{wt} \%$ ) and carboxylated Multi-walled carbon nanotubes (outer diameter $8-15 \mathrm{~nm}$, length $\sim 50 \mu \mathrm{m}$, purity $\geq 99.9 \mathrm{wt} \%$ ) were used as solid phase for chiral resolution. Fig. 1 shows the transmission electron miscroscopy image of aligned multi-walled carbon nanotubes.

\section{Experimentals}

The MWCNTs (outer diameter $<8 \mathrm{~nm}$, length $10-30 \mu \mathrm{m}$, purity $\geq 95 \mathrm{wt} \%$ ), aligned Multi-walled carbon nanotubes (outer diameter $10-20 \mathrm{~nm}$, length $30-100 \mu \mathrm{m}$, purity $\geq 95 \mathrm{wt} \%$ ), amino functionalized Multi-walled carbon nanotubes (outer diameter $8-15 \mathrm{~nm}$, length $\sim 50 \mu \mathrm{m}$, purity $\geq 95 \mathrm{wt} \%$ ) and carboxylated Multi-walled carbon nanotubes (outer diameter 8-15 nm, length $\sim 50$ $\mu \mathrm{m}$, purity $\geq 99.9 \mathrm{wt} \%$ ) were all purchased from Nanjing JCNANO Tech Co., LTD.; D-tartaric acid was obtained from U.S. Woke Reagents Co., Ltd; Naptalam was bought from Aladin Reagents Co., LTD.

Impregnated thin-layer plate $(5 \mathrm{~cm} \times 10 \mathrm{~cm})$ were prepared by spreading a slurry of silica gel GF254 (1.5 g), $0.02 \mathrm{~g}$ functionalized multi-walled carbon nanotubes and sodium carboxymethylcellulose solution $(4 \mathrm{~mL}, 0.5 \mathrm{wt} \%)$ with an automatic thin layer applicator and then immersed in aqueous D-tartaric acid solution (10-3 mol/L) for 5 minutes. The plates were air dried firstly, then activated in vacuum under $110^{\circ} \mathrm{C}$ for $1.5 \mathrm{hrs}$, and stored in a vacuum desiccator for chromatographic use.

The solution of naptalam solution $(0.50 \mathrm{~g} / \mathrm{L})$ was fetched with glass spotting capillary, and was spotted at $1 \mathrm{~cm}$ above the bottom edge of the plate, then was putted into P-1 TLC developing chamber to develop under ambient temperature through using the solution of cyclohexane-alcohol as the developer. After developing, it was dried in infrared desiccator, and the red spot was applied in ZT-1 UV analyzer under 365nm ultraviolet. The relative RF value is the ratio of RF value of two naptalam enantiomers.

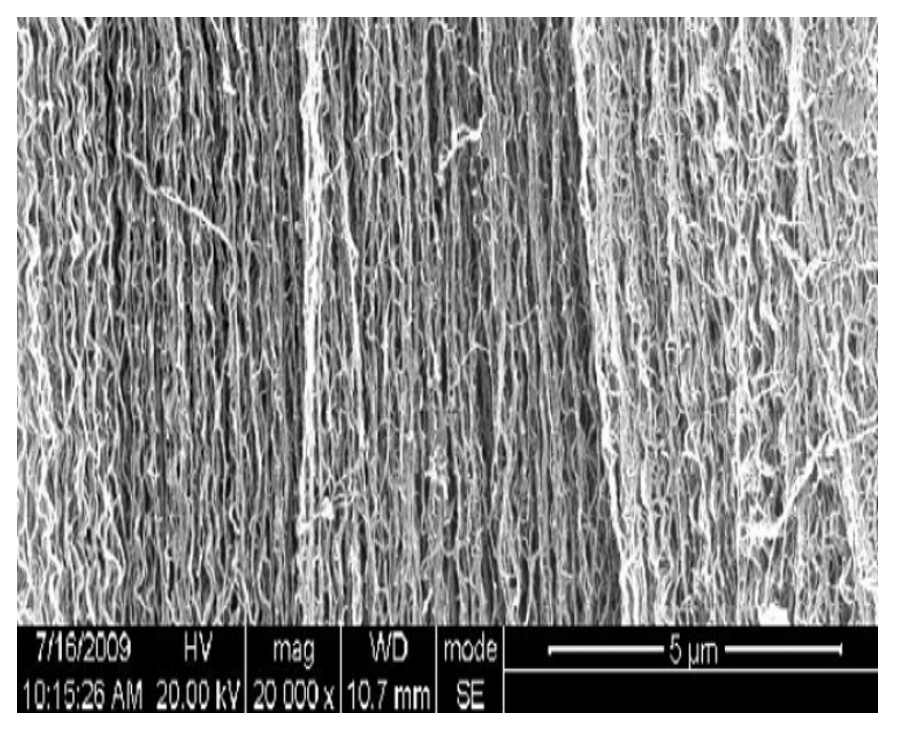

Fig. 1. High resolution transmission electron miscroscopy image of aligned multi-walled carbon nanotubes.

\section{Result and Discussion}

\section{Influence of different kind of alcohol on the resolution.}

Resolutions of naptalam using MWCNTs impregnated thin-layer chromatography with D-tartaric acid as chiral selector and cyclohexane-alcohol solvent mixture 70:30 as developers were investigated. The alcohols included methanol, ethanol, isopropanol, n-butanol, sec-butyl alcohol, 
tert-butyl alcohol, n-amyl alcohol, n-hexyl alcohol, n-heptyl alcohol and n-octyl alcohol. The effect of different kind of alcohol on the resolution using MWCNTs -impregnated TLC is shown in Tab. 1. The results indicated that resolution could be carried out by using cyclohexane: butanol as developers, the relative value of RF was between 2.15 and 2.56, the relative value of RF of solvent mixture of cyclohexane: n-butyl alcohol was the largest, and the effect of resolution was the better.

Tab.1 The effect of different kind of alcohol on the resolution using MWCNTs -impregnated TLC

\begin{tabular}{cccc}
\hline $\begin{array}{c}\text { cyclohexane : alcohol } \\
(\mathbf{v} / \mathbf{v}=\mathbf{5 0}: \mathbf{5 0})\end{array}$ & $\mathbf{R}_{\mathbf{f}-\mathbf{1}}$ & $\mathbf{R}_{\mathbf{f}-\mathbf{2}}$ & $\begin{array}{c}\text { Relative value } \\
\text { of } \mathbf{R F}\end{array}$ \\
\hline cyclohexane: methanol & 0.15 & 0.26 & 1.73 \\
cyclohexane: ethanol & 0.22 & 0.28 & 1.28 \\
cyclohexane: isopropanol & 0.18 & 0.30 & 1.67 \\
cyclohexane: n-butanol & 0.16 & 0.41 & 2.56 \\
cyclohexane: 2-butanol & 0.22 & 0.47 & 2.15 \\
cyclohexane: tert-butanol & 0.20 & 0.45 & 2.26 \\
cyclohexane: n-pentanol & 0.43 & - & - \\
cyclohexane: n-hexanol & 0.42 & - & - \\
cyclohexane: n-heptanol & 0.37 & - & - \\
cyclohexane: n-octanol & 0.35 & - & - \\
\hline
\end{tabular}

\section{Influence of the percentage of n-butanol on the resolution.}

Resolutions of naptalam using MWCNTs impregnated thin-layer chromatography with D-tartaric acid as chiral selector and and influences of different percentage of n-butanol and cyclohexane were investigated. The effect of the percentage of $n$-butanol on the resolution using MWCNTs -impregnated TLC is shown in Tab. 2. The results indicated that resolution could be carried out only when the percentage of cyclohexane was more than $40 \%$, the values of $\mathrm{R}$ were more than 1.4. Comparing TLC chromatogram pictures in the experimental result, it was found that under the condition of $\mathrm{V}($ cyclohexane $): \mathrm{V}(\mathrm{n}$-butanol $)=30: 70$, the sizes of spots were even, and there is no phenomenon of tail and the effect of resolution was the best.

\section{Influence of buffer solutions on resolution.}

Buffer solutions can improve the effect of resolution generally, and among them the buffer solution of Na2HPO4 is the most used. The result of resolution of naptalam using solvent mixture cyclohexane -n-butanol as developer indicated that there was phenomenon of slight tailing of the sample spots. Considered that naptalam has the character of acidity, the buffer solution of Na2HPO4(0.1M, $\mathrm{pH}=3)$ was confected as tail inhibitor and was added into the developer, while its proportion changed from $0.2 \% \sim 2.0 \%$, it was found that it couldn't improve the phenomenon of tail and it influenced the effect of resolution seriously, and while the separate degree was 0 , the resolution couldn't be carried out. We select the condition of not adding buffer solution in the experiment because of the slight tail.

\section{Influence of various functionalized MWCNTs on the resolution.}

Resolutions of naptalam using various functionalized MWCNTs impregnated thin-layer chromatography with D-tartaric acid as chiral selector and cyclohexane-alcohol solvent mixture 70:30 as developers were investigated. The functionalized MWCNTs include MWCNTs, aligned MWCNTs, amino functionalized and carboxylated MWCNTs. The effect of the various functionalized MWCNTs on the resolution on the resolution using MWCNTs -impregnated TLC is 
shown in Tab. 3 .

Tab.2 The percentage of n-butanol on the resolution using MWCNTs -impregnated TLC

\begin{tabular}{cccc}
\hline $\begin{array}{c}\text { cyclohexane: } \\
\text { n-butanol (v/v) }\end{array}$ & $\mathbf{R}_{\mathbf{f}-\mathbf{1}}$ & $\mathbf{R}_{\mathbf{f}-\mathbf{2}}$ & $\begin{array}{c}\text { Relative } \\
\text { value of RF }\end{array}$ \\
\hline $100: 0$ & 0.13 & 0.43 & 3.31 \\
$90: 10$ & 0.18 & 0.55 & 3.05 \\
$80: 20$ & 0.25 & 0.56 & 2.24 \\
$70: 30$ & 0.16 & 0.41 & 2.56 \\
$60: 40$ & 0.21 & 0.56 & 2.67 \\
$50: 50$ & 0.28 & 0.40 & 1.43 \\
$40: 60$ & 0.27 & 0.68 & 2.52 \\
$30: 70$ & 0.28 & - & - \\
$20: 80$ & 0.72 & - & - \\
$10: 90$ & 0.64 & - & - \\
$0: 100$ & 0.39 & - & - \\
\hline
\end{tabular}

Tab.3 The effect of the various functionalized MWCNTs on the resolution using MWCNTs -impregnated TLC

\begin{tabular}{cccc}
\hline $\begin{array}{c}\text { various functionalized } \\
\text { MWCNTs }\end{array}$ & $\mathbf{R}_{\mathbf{f}-1}$ & $\mathbf{R}_{\mathbf{f}-2}$ & $\begin{array}{c}\text { Relative value } \\
\text { of } \mathbf{R F}\end{array}$ \\
\hline MWCNTs & 0.16 & 0.41 & 2.56 \\
aligned MWCNTs & 0.24 & 0.32 & 1.33 \\
$\begin{array}{c}\text { amino functionalized } \\
\text { MWCNTs }\end{array}$ & 0.19 & 0.82 & 4.32 \\
carboxylated MWCNTs & 0.11 & 0.36 & 3.27 \\
\hline
\end{tabular}

The results indicated that good resolution of naptalam enantiomer can be achieved by MWCNTs impregnated TLC. While using functionalized MWCNTs such as aligned MWCNTs and MWCNTs, a poor resolution achieved may be due to the strong interaction between the MWCNTs and the enantiomers. In contrast, the relative RF value of amino functionalized MWCNTs impregnated TLC was significantly higher than other functionalized MWCNTs impregnated TLC. This is because that the force difference of two naptalam enantiomers with the stationary phase expanded due to the carboxyl modification of the carbon nanotubes.

\section{Conclusion}

Effective chiral resolution of naptalam was achieved by functionalized multi-walled carbon nanotubes impregnated thin-lay chromatography plates. D-tartaric acid was used as chiral selector and cyclohexane-alcohol solvent mixture was used as developers.

Under the condition of $\mathrm{V}($ cyclohexane $): \mathrm{V}(\mathrm{n}$-butanol $)=30: 70$, the sizes of spots were even, and there is no phenomenon of tail and the effect of resolution was the best. 
The results indicated that chiral naptalam can be resoluted well by thin-lay chromatography impregnated with various functionalized multi-walled carbon nanotubes such as MWCNTs, aligned MWCNTs, amino functionalized MWCNTs and carboxylated MWCNTs. And the relative RF value of amino functionalized multi-walled carbon nanotubes impregnated thin-lay chromatography was significantly higher than that of other types of multi-walled carbon nanotubes impregnated thin-lay chromatography. The relative RF value of amino multi-walled carbon nanotubes mediated thin-lay chromatography was 4.32 . We hope that multi-walled carbon nanotubes/D-tartaric acid impregnated thin-lay chromatography could be useful to the chromatographer and pharmaceutical industries.

\section{Acknowledgement}

This work was supported by the National Natural Science Foundation of China (No.21366011, $61361002,21276071,21461007)$ and the open fund of master pilot construction of chemical subject (No.HXZ1302).

\section{References}

[1] Otto L. Hoffmann and Allen E. Smith. A New Group of Plant Growth Regulators. Science. 109(2841): 588. (1949)

[2] Shingo Harada, Satoru Kuwano, Yousuke Yamaoka, Ken-ichi Yamada, Kiyosei Takasu. Kinetic Resolution of Secondary Alcohols Catalyzed by Chiral Phosphoric Acids. Angewandte Chemie International Edition. 2013, 52 (39): 10227-10230

[3] Nobar Jalili, Narjes Ansari, Francesc Viñes, Francesc Illas, Fariba Nazari. Ionic Liquid Chiral Resolution: Methyl 2-Ammonium Chloride Propanoate on Al(854)S Surface. J. Phys. Chem. C, 2014, 118 (3): 1568-1575

[4] Toshiyuki Kida, Takuya Iwamoto, Haruyasu Asahara, Tomoaki Hinoue, Mitsuru Akashi. Chiral Recognition and Kinetic Resolution of Aromatic Amines via Supramolecular Chiral Nanocapsules in Nonpolar Solvents. J. Am. Chem. Soc., 2013, 135 (9): 3371-3374

[5] Boelo Schuur, Bastiaan J. V. Verkuijl, Adriaan J. Minnaard, Johannes G. de Vries, Hero J. Heeres and Ben L. Feringa. Chiral separation by enantioselective liquid-liquid extraction. Org. Biomol. Chem., 2011,9, 36-51

[6] Xuan Kuang, Yu Ma, Hao Su, Jine Zhang, Yu-Bin Dong, Bo Tang. High-Performance Liquid Chromatographic Enantioseparation of Racemic Drugs Based on Homochiral Metal-Organic Framework. Anal. Chem., 2014, 86 (2): 1277-1281

[7] Li, L., Zhou, S. S., Zhao, M. R., Zhang, A. P., Peng, H., Tan, X. S., Lin, C. M., He, H. W., Separation and aquatic toxicity of enantiomers of 1-(substituted phenoxyacetoxy)alkylphosphonate herbicides, Chirality, 20, 130-138. (2008)

[8] Lin, K. D., Xu, C., Zhou, S. S., Liu, W. P., Gan, J., Enantiomeric separation of imidazolinone herbicides using chiral high-performance liquid chromatography, Chirality, 19, 171-178. (2007)

[9] Na, N.; Cui, X.; De Beer, T.; Liu, T.; Tang, T.; Sajid, M.; Ouyang, J. The use of silica nanoparticles for gas chromatographic separation. Journal of Chromatography A, 1218, 4552-4558. (2011)

[10]Noaman Ul-Haq and Joong Kon Park. Effect of Template on Chiral Separation of Phenylalanine using Molecularly Imprinted Membrane in Aqueous Medium. J. Chem. Soc. Pak., 36( 4), 606-613,(2014)

[11]Huang D.S., Yu J.G., Liu W., et al. Multi-Walled Carbon Nanotubes Mediated Thin-Layer Chromatographic Enantioseparation of Ofloxacin. Current Nanoscience, 9(1), 139-140. (2013) 
[12]Mohammad Afrasiabi, Shokat Kianipour, Zeinab Rezayati Zad, Ali Babaei. Application of Multi-Walled Carbon Nanotubes Modified Glassy Carbon Electrode for Determination of Mefenamic Acid in Pharmaceutical Preparations and Biological Fluids. J. Chem. Soc. Pak., 35(4), 1113-1121, 2013

[13]Yonggang $\mathrm{Xu}$, Deyuan Zhang, Jun Cai, Liming Yuan and Wenqiang Zhang. Effects of Multi-walled Carbon Nanotubes on the Electromagnetic Absorbing Characteristics of Composites Filled with Carbonyl Iron Particles. J. Mater. Sci. Technol., 28(1), 34-40. (2012)

[14]Chao Luo, Rongyan Wei, Dan Guo, Shengfang Zhang, Shiqiang Yan. Adsorption behavior of $\mathrm{MnO} 2$ functionalized multi-walled carbon nanotubes for the removal of cadmium from aqueous solutions. Chemical Engineering Journal. 225, 406-415. (2013) 